\title{
Flow analysis in a channel with flexible vegetation using double-averaging method
}

\author{
Maurizio RIGHETTI \\ Department of Civil and Environmental Engineering, University of Trento, \\ Trento, Italy; e-mail: maurizio.righetti@ing.unitn.it
}

\begin{abstract}
The paper addresses the problem of the resistance due to vegetation in an open channel flow, characterized by partially and fully submerged vegetation formed by colonies of bushes. The flow is characterized by significant spatial variations of velocity between vertical profiles that make the traditional approach based on time averaging of turbulent fluctuations inconvenient. A more useful procedure, based on time and spatial averaging (Double-Averaging Method) is applied for the flow field analysis and characterization. The vertical distribution of mean velocity and turbulent stresses at different spatial locations has been measured with a 3D Acoustic Doppler Velocimeter (ADV) for two different vegetation densities where fully submerged real bushes (salix pentandra) have been used. Velocity measurements were completed together with the measurements of drag exerted on the flow by bushes at different flow depths. The analysis of velocity measurements allows depicting the fundamental characteristics of both the mean flow field and turbulence. The experimental data show that the contribution of form-induced stresses to the momentum balance cannot be neglected. The mean velocity profiles and the spatially averaged turbulent intensity profiles allow inferring that the vegetation density is a driving parameter for the development of a mixing layer at the canopy top in the case of submerged vegetation. Moreover, the net upward turbulent momentum flux, evaluated with the methodology proposed by $\mathrm{Lu}$ and Willmarth (1973), appears to be damped for increased vegetation density; this finding can rationally explain the reduction of the suspended sediment transport capacity typically observed in free surface flows over a vegetated bed.
\end{abstract}

Key words: vegetated channels, turbulence, Double-Averaging Method.

(C) 2008 Institute of Geophysics, Polish Academy of Sciences 


\section{INTRODUCTION}

The importance of vegetation as a factor of improvement of the environmental value of rivers is widely recognized and the use of living bushes in restoring regulated rivers and mountain streams is encouraged. Therefore, hydrophilous riparian plants, like willows, are not only naturally present on the banks and floodplains and sporadically also in riverbeds, but are often used as living material for banks preservation as well. The proper maintenance practices tend to preserve the plants elasticity characteristics (e.g., by limiting plant growth by means of periodical lopping), in order to allow vegetation to promptly bend and adapt to floods and to assume a more hydrodynamic shape. Therefore, the problem of hydrodynamic behaviour of flexible bushes fully submerged in the flow is quite common in hydraulics of both natural and restored streams. Willow height is of same order of magnitude as the flow depth, causing drag and energy losses. The resistance that willows create to the flow can be evaluated as the combined effect of the hydrodynamic drag of single plants (Petryk and Bosmanijan 1975), according to the equation:

$$
F=C_{D} A_{P} \rho \frac{U^{2}}{2},
$$

where $F$ is the drag force exerted on a single plant, $A_{p}$ is the area of the plant projected in streamwise direction (frontal area in the following), $\rho$ is the water density, $C_{D}$ is the drag coefficient of a single plant, and $U$ is the mean flow velocity. The plant drag per unit bed surface area and unit plant height can be evaluated with the following equation:

$$
f_{V}=n F / h_{P},
$$

where $n$ is the number of plants per unit bed surface area and $h_{P}$ is the plant height. Therefore, the net bed shear stress $\tau_{b}^{\prime}$ is given by the equation:

$$
\tau_{b}^{\prime}=\rho g R_{h} S_{b}-f_{V} h_{P} \approx \rho g h S_{b}-f_{V} h_{P},
$$

where $g$ is the gravitational acceleration, $R_{h}$ is the hydraulic radius, $S_{b}$ is the bed slope, and $h$ is the flow depth.

The drag can be included into rational formulations for the evaluation of the flow resistance (see e.g., Ming and Shen 1973, Petryk 1975, Righetti and Armanini 2002). The physical conditions of riverine vegetation, e.g., plant species, age and vegetation density, are extremely variable, which strongly affects the resistance coefficient and has to be properly taken into account in the hydraulic calculations. Moreover, the flow velocities are usually very low in vegetated areas or wetlands compared to rivers, so turbulent characteristics become important because of the sediment deposition and erosion in the vegetation zone (Lopez and Garcia 2001, Nepf and Vivoni 1999). The presence of plants strongly affects the structure of the turbulent flow through 
and above the vegetation: the straining of motion due to stems and the wake turbulence they generate, significantly affect the turbulent energy dynamics (Nepf 1999). The effect of fully submerged vegetation on the flow field can be interpreted as a low relative submergence roughness, characterized by spatial variability of the length scales of the same order of magnitude as the flow depth. In this case, a proper approach derives from a double-averaging procedure on the classical Navier-Stokes equations, where the classical timeaveraged Reynolds equations should be supplemented by spatial area averaging procedure in the plane parallel to the average bed. A similar procedure has been previously applied to terrestrial canopies (Wilson and Shaw 1977, see Finnigan 2000) and can be considered to be a reference framework also for vegetated rivers - even though differences exist in the turbulence structure of the unconfined flow field above canopies and confined free surface flows (Nepf and Vivoni 1999) - as well as to rough bed open channel flows with small relative submergence (Nikora et al. 2001).

Studies on terrestrial canopies (Raupach et al. 1996) and experimental observation in laboratory open channel flows (Ikeda and Kanazawa 1996, Carollo et al. 2002, Ghisalberti and Nepf 2002, Ghisalberti and Nepf 2004) demonstrate that the flow within and above vegetation shows a characteristic $S$ shaped velocity profile, thus a mixing layer analogy seems to be more appropriate than a boundary layer analogy for its interpretation. These studies have been mainly based on laboratory experiments conducted with artificial models of vegetation, such as rigid cylinders or plastic strips, or with small scale flexible plants, such as macrophytes, but flows over riparian willows have not been studied yet. In the literature, studies of real vegetation generally focused on wetland's plants (e.g., sedges), marshes, or on rigid plants, usually schematised as cylinders, or groups of cylinders, in the laboratory tests. Indeed, as a matter of fact, the plants flexibility increases the complexity of both the vegetation resistance and the turbulent flow field characterization. Experiments concerning real flexible riverine plants, such as salix, are relatively rare and mainly refer to the drag evaluation for isolated plants (Oplatka 1998, Armanini et al. 2005) or on group of plants partially or totally submerged by the flow (Fathi-Maghadam and Kouwen 1997, Freeman et al. 2000, Jarvela 2002).

What comes out from the data in literature is the difficulty to extend the laboratory results to the field (as well as paucity of data concerning real flexible bushes). Indeed, living materials naturally have highly variable properties even within one single species; therefore, the extension of the laboratory results to field scale is subject to a high degree of uncertainty.

In this paper, measurements of flow velocity and hydrodynamic forces acting on real willows, fixed at the bottom of a laboratory channel, are presented. The aim of the present study is to improve knowledge of the interac- 
tion between the flow and real flexible bushes, by analyzing the main features of the turbulent flow field in the frame of the Double-Averaging Method. Real bushes have been studied both in partial and in fully submerged conditions, at two different densities. The willows used in the experiments were Salix Pentandra, a kind of plants mostly used in restoration works along mountain streams. Drag force measurements have been made during all experiments, with detailed velocity measurements in two experiments with fully submerged plants. The force measurements allow to quantify the relative contribution to the flow resistance of the bed friction and plants drag, for the different plants densities. Moreover, for the two runs with plants fully submerged by the flow, the main features of the turbulent flow field is analysed in the frame of the Double-Averaging Method.

\section{EXPERIMENTAL INSTALLATION AND MEASUREMENT TECHNIQUE}

Experiments were conducted at the Experimental Centre for Hydraulic Models of Consorzio Venezia Nuova, in Voltabarozzo (Padova-Italy). The laboratory flume used in the tests is $150 \mathrm{~m}$ long, $2 \mathrm{~m}$ wide, $2 \mathrm{~m}$ deep and the bed and the walls are of concrete, except for a length of $12 \mathrm{~m}$ where one of the banks is made of glass. Through this glass windows, the plants bending has been registered by a digital photo camera and analyzed with image analysis techniques. The final section of the flume ends with a rectangular adjustable weir. The water flows through a closed circuit by means of a group of four pumps, up to a maximum discharge of $1.57 \mathrm{~m}^{3} \mathrm{~s}^{-1}$. The channel is equipped with a double-track carriage which moves all along the flume. The tracks rest at the top of the concrete lateral walls. The carriage was used for the ADV velocity measurements, as explained in the following. The discharge is measured by means of an electromagnetic flow meter. The slope of the channel bed $S_{b}$ is $0.18 \%$. The downstream part of the flume was uniformly covered with salix bushes for a length of $45 \mathrm{~m}$. The bushes had heights $h_{P}$ ranging between 0.6 and $0.7 \mathrm{~m}$. The bushes were placed at the bed in staggered configuration, with two different densities, named sparse and dense configuration in the following.

The geometrical characteristics of the plants positioning is reported in Figs. 1 and 2. In the sparse configuration, 1.1 bushes per square meter were placed, while the dense configuration is characterised by the presence of 2.5 bushes per square meter. Figure 1 also shows the position of the force transducer used for plant drag measurements.

Uniform inlet conditions were achieved using a perforated brick wall as a flow straightener to eliminate swirl. Just upstream the $45 \mathrm{~m}$ long reach with bushes, two rows of rigid wood sticks, $60 \mathrm{~cm}$ high, were placed, in order to minimize the bending of the first rows of salix invested by the flow. 

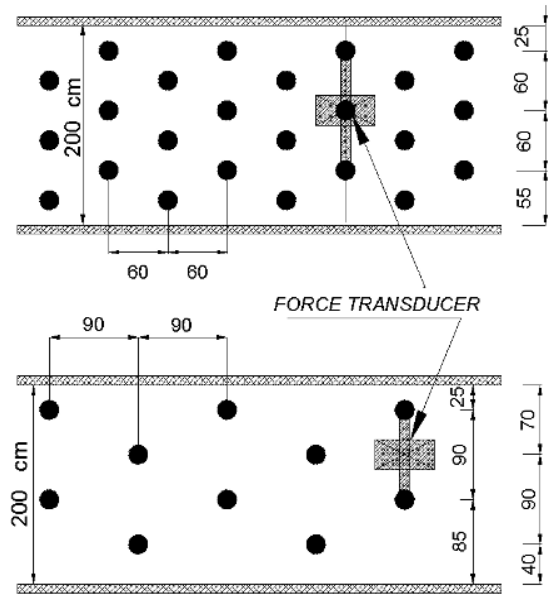

Fig. 1. Sketch of the plan view of the open channel with the two vegetation densities considered.

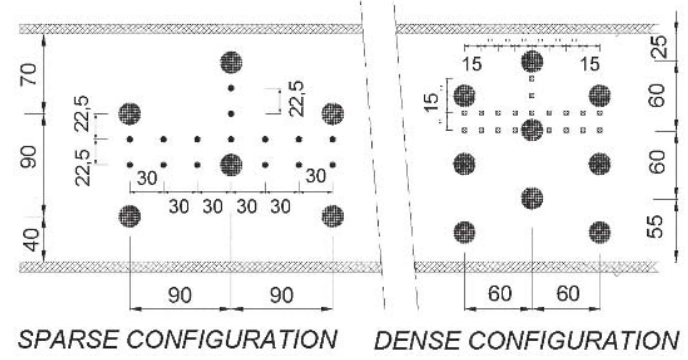

Fig. 2. Location of the verticals along which the ADV measurements were performed in the dense and sparse configurations.

For each discharge, the water level was measured all along the vegetated area and for every flow condition the formation of three reaches was observed. A transition profile was observed to develop in the first $10-15 \mathrm{~m}$ of the vegetated area, then a constant 20-27 m long flow depth region followed and finally a 5-10 m long, mild accelerating M3 profile developed. The length of each reach depends on the discharge values and on the deflected plants height-flow depth ratio $h_{P}^{\prime} / h$. Therefore, for each discharge the water level at the downstream end of the flume was checked and adjusted by means of a sluice gate, in order to minimize the development of the M3 profile. The measuring zone was located $33 \mathrm{~m}$ downstream from the beginning of the vegetated area. Therefore, the flow conditions were not completely uniform all along the vegetated area, but it can reasonably be assumed that the uniform (or very close to uniform) flow conditions were achieved in the measuring zone, adjusting the sluice gate. 
The selected plants were similar in height and also in shape, diameter of the main trunk, and principal and second order branches. Moreover, for the plants placed on the force transducer, the variation of the plants frontal area $A$ with the distance $z$ to the bed has been measured by means of image analysis techniques. Therefore, this area $A(z)$ represents the frontal area of the plant that would be submerged by a flow having depth $z$. It has to be remarked that, like in Armanini et al. (2005), only the trunk and the main branches have been considered for the frontal area definition. The normalized plant frontal area, that is, the ratio of $A(z)$ to $A_{p}=A\left(z=h_{p}\right)$, versus the relative height $z / h_{p}$, is plotted in Fig. 3 and compared with analogous measurements performed on willows sampled by plant cutting along the banks of some restored mountain streams and ranging between 1.5 and $3.5 \mathrm{~m}$ in height (Nobile 2007). It can be noted that the similitude between the laboratory and field willows is quite good.

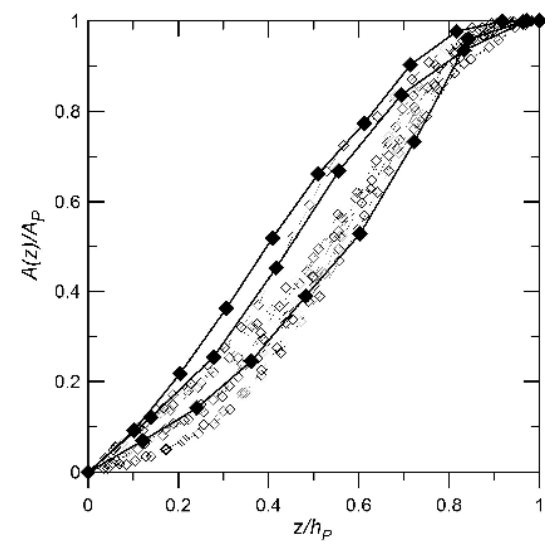

Fig. 3. Cumulative frontal area as a function of plants height. $-\diamond-$ real salix, $3 \mathrm{~m}$ tall, sampled along the banks of mountain streams in Trentino Region (Nobile 2007), $\rightarrow-$ willows sampled in the laboratory channel.

A detailed analysis of the distribution of plant biomass with height and of plant density - defined as the number of plants per unit surface of the bed - has been performed for naturalized torrents. This analysis had the aim of reproducing the same vegetation density and plant biomass distribution at laboratory scale as sampled in the field (Nobile 2007). The results of the analysis will be discussed in the next section. The sparse vegetation density was chosen to be similar to that measured on the banks of some mountain streams. For each configuration a series of three runs was performed, for which the discharge was ranging between about 0.4 and $1.5 \mathrm{~m}^{3} \mathrm{~s}^{-1}$, as reported in Table I, where also the ratio of deflected to undeflected plants height, $h_{P}^{\prime} / h_{p}$, is reported. During each run, also the force exerted by the flow on a sample of bushes (three bushes for the dense configuration and 
Table 1

Main features of the runs performed

\begin{tabular}{|c|c|c|c|c|c|c|c|c|c|}
\hline \multicolumn{4}{|c|}{ Sparse configuration $\left(h_{P}=0.70 \mathrm{~m}\right)$} & \multicolumn{5}{|c|}{ Dense configuration $\left(h_{P}=0.70 \mathrm{~m}\right)$} \\
\hline $\begin{array}{c}Q \\
{\left[\mathrm{~m}^{3} / \mathrm{s}\right]}\end{array}$ & $\begin{array}{c}Q / A \\
{[\mathrm{~m} / \mathrm{s}]}\end{array}$ & $\begin{array}{c}H \\
{[\mathrm{~m}]}\end{array}$ & $\begin{array}{c}h_{P}^{\prime} \\
{[\mathrm{m}]}\end{array}$ & $h_{P}^{\prime} / h$ & $\begin{array}{c}Q \\
{\left[\mathrm{~m}^{3} / \mathrm{s}\right]}\end{array}$ & $\begin{array}{c}Q / A \\
{[\mathrm{~m} / \mathrm{s}]}\end{array}$ & $\begin{array}{c}H \\
{[\mathrm{~m}]}\end{array}$ & $\begin{array}{c}h_{P}^{\prime} \\
{[\mathrm{m}]}\end{array}$ & $h_{P}^{\prime} / h$ \\
\hline 0.556 & 0.524 & 0.53 & 0.69 & 0.99 & 0.422 & 0.402 & 0.525 & 0.69 & 0.99 \\
\hline 0.867 & 0.647 & 0.67 & 0.67 & 0.96 & 0.711 & 0.531 & 0.67 & 0.67 & 0.96 \\
\hline $\begin{array}{c}1.533 \\
*\end{array}$ & $\begin{array}{c}0.824 \\
*\end{array}$ & $\begin{array}{c}0.93 \\
*\end{array}$ & $\begin{array}{c}0.45 \\
*\end{array}$ & $\begin{array}{c}0.65 \\
*\end{array}$ & $\begin{array}{c}1.311 \\
*\end{array}$ & $\begin{array}{c}0.728 \\
*\end{array}$ & $\begin{array}{c}0.90 \\
*\end{array}$ & $\begin{array}{c}0.58 \\
*\end{array}$ & $\begin{array}{c}0.83 \\
*\end{array}$ \\
\hline
\end{tabular}

* denotes the two runs for which also the velocity measurements were performed

two bushes for the sparse) was measured by means of the submerged force transducer. The aim of these measurements was twofold: the characterization of the plants drag evolution at increasing water discharge and thus for increasing bending, and also evaluation of the relative importance of plant drag to bed friction resistance. The force transducer was made up of two steel plates $(30 \times 15 \mathrm{~cm})$ bound together by four aluminium foils at which eight strain gauges were stuck in order to form four Wheatsone bridges. The willows were made perfectly integral to the superior plate of the force transducer. The Weathsone bridges allowed to measure the three components of strength and the bending moment produced by the force acting on the willows along the main flow direction. The circuits were calibrated and carefully waterproofed to allow the use of this device in completely submerged conditions. The force transducer was placed at the bottom of the flume within the measuring zone (see Fig. 1). For further details about the flume and the force transducer, the reader can refer to Armanini et al. (2005).

As reported in Table 1, in four of the six runs the plants were partially submerged by the flow. However, for two runs, one for the dense configuration and one for the sparse, the bending of the plants makes them to be fully submerged by the flow, and the height of the bowed vegetation $h_{P}^{\prime}$ was substantially less than the flow depth $h$. For these two runs, 15 velocity profiles for the sparse configuration and 19 velocity profiles for the dense configuration were measured. The exact locations of the measured profiles are shown in Fig. 2. A 3-D acoustic Doppler velocimeter (Sontek ADV) was used to measure the streamwise $(u)$, transverse $(v)$, and vertical $(w)$ velocity components in 20 points along each of the verticals. Therefore, the data at 300 measurement points for the sparse configuration and at 380 measurement points for the dense configuration were collected. The analysis of these results in the DAM framework deserves particular attention and will be discussed in the next sections. 


\section{ANALYTICAL METHOD}

Fully submerged willows act on the flow field as a low relative submergence roughness, characterized by spatial variability of the length scales of the same order of magnitude as the flow depth. In this case, a proper approach derives from a double-averaging procedure of the traditional Navier-Stokes equations, where the time-averaged Reynolds equations should be supplemented by spatial area averaging procedure in the plane parallel to the average bed (see, e.g., Wilson and Shaw 1977, Finnigan 2000, Nikora et al. 2001, Righetti and Armanini 2002). Consider the case of a 2D, steady, uniform, spatially averaged flow over a fully submerged vegetation, where $x$ is the longitudinal direction, $y$ is the transversal direction and $z$ is the vertical coordinate. Applying the double-averaging technique (in time and in the space, that is in the $x, y$ plane) to the Navier-Stokes equations, and neglecting viscous terms, two sets of differential equations are obtained:

$$
\begin{gathered}
g S_{b}-\left\langle\frac{\partial \tilde{p}}{\partial x}\right\rangle-\frac{1}{\phi} \frac{\partial \phi\left\langle\overline{u^{\prime} w^{\prime}}\right\rangle}{\partial z}-\frac{1}{\phi} \frac{\partial \phi\langle\tilde{u} \tilde{w}\rangle}{\partial z}=0, \\
g+\frac{1}{\rho} \frac{\partial\langle\bar{p}\rangle}{\partial z}+\frac{1}{\rho}\left\langle\frac{\partial \tilde{p}}{\partial z}\right\rangle+\frac{1}{\phi} \frac{\partial \phi\left\langle\overline{w^{\prime 2}}\right\rangle}{\partial z}+\frac{1}{\phi} \frac{\partial \phi\left\langle\tilde{w}^{2}\right\rangle}{\partial z}=0,
\end{gathered}
$$

where $u, v, w$ are the instantaneous velocity components along the three coordinate axes $x, y, z$, respectively, $p$ is the pressure. The prime $\left(X^{\prime}\right)$ represents the point temporal fluctuation of $X$, the brackets $(\langle X\rangle)$ and the linear overbar $(\bar{X})$ refer, respectively, to the spatial averaging along $(x, y)$ plane and to time-averaging, $S_{b}$ is the bed slope, $g$ is the gravitational acceleration, $\Phi$ is the combined spatial and temporal porosity of vegetation (if present) in the $(x, y)$ layer where the space averaging is performed, that is, the ratio between the area occupied by fluid and the total area of the averaging region in the $x, y$ plane at level $z$ : its values range between 0 and 1 in the vegetated region. Wavy overbars denote the difference between time averaged and doubleaveraged values $(\tilde{X}=\bar{X}-\langle\bar{X}\rangle)$ and represents the form-induced stresses due to spatial averaging of the Navier-Stokes equations. Equation (4) represents the momentum balance in the longitudinal direction, while eq. (5) the balance in the vertical one. The mean velocity and turbulence measurements, till the third-order turbulence moments, carried out during the experiments were analyzed in the frame of the double-averaged momentum equations; the main features will be presented in the next section.

\section{RESULTS AND DISCUSSION}

\section{Porosity of vegetation and plants bending}

An analysis of the distribution of plants biomass with height and of plants density - defined as the number of plants per unit surface of the bed - allows 


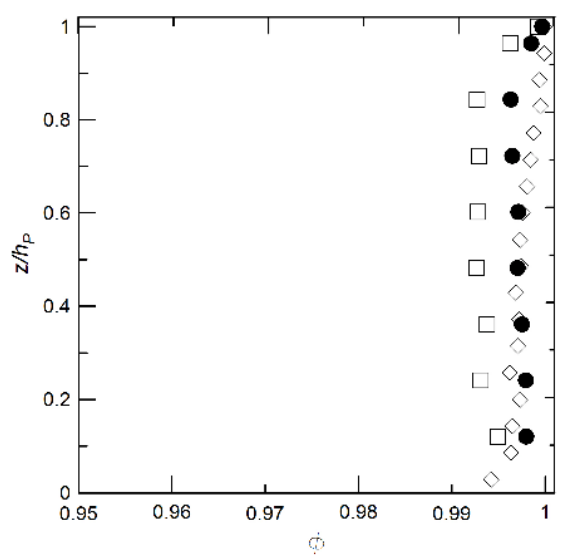

Fig. 4. Porosity of vegetation. $\diamond$ measurements on real salix, sampled along the banks of mountain streams in Trentino Region (Italy); $\bullet$ willows sampled in the laboratory channel, sparse configuration; $\square$ willows sampled in the laboratory channel, dense configuration.

to assess the influence of vegetation porosity function on the balance equations. Figure 4 shows the porosity of vegetation $\Phi(z)$ evaluated on the banks of naturalized torrents (Nobile 2007) and as reproduced in the laboratory experiment for the sparse and dense configurations. In all the cases, porosity has been evaluated following the definition inferred in eqs. (4) and (5), that is, evaluating the area occupied by the trunks and main branches of plants over an averaging region containing a number of plants, so that $\Phi(z)$ is the porosity of the area used for spatial averaging and includes in its definition also the number of plants per unit surface of the averaging region. Moreover, it is worth of note that the vegetation porosity tends to assume values almost constant and very close to unity all along the plants height; therefore, despite the fact that the density of plants and so the porosity of vegetation could surely affect the flow resistance and the turbulence structure, the values of the parameter $\Phi$ can be assumed almost constant and substantially equal to one in eqs. (4) and (5), thus leading to an undoubted simplification in the balance equations.

Finally, in Fig. 5 the typical trunk bending of two laboratory plants belonging to the dense and sparse configurations are reported for different mean flow velocities. The data in Fig. 5 refers to the runs reported in Table 1, for which the corresponding bending has been estimated by image analysis techniques. The deflection of the plants is significant, inducing a significant reduction of the thickness of the vegetated layer during the flows. The plot shows that sparse configuration tends to exhibit lower flow depths and more deflected plants with respect to the dense configuration, for the same disdischarge. Therefore, it can be argued that for a given discharge the drag acted by each plant increases for decreasing plant density thus giving a more 


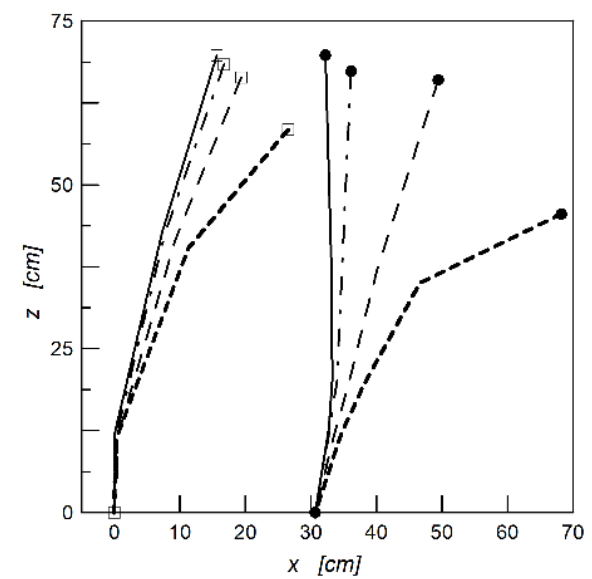

Fig. 5. Typical trunk bending for a willow belonging to the dense and sparse configurations, at increasing discharges. Dense configuration: $\square-Q / A=0$, — च- $Q / A=0.40 \mathrm{~m}^{3} \mathrm{~s}^{-1}, h=0.52 \mathrm{~m}, \quad$ - в - $Q / A=0.53 \mathrm{~m}^{3} \mathrm{~s}^{-1}, h=0.67 \mathrm{~m}$, --в-- $Q / A=0.728 \mathrm{~m}^{3} \mathrm{~s}^{-1}, h=0.90 \mathrm{~m}$. Sparse configuration: $\longrightarrow-\longrightarrow / A=0$, •- $Q / A=0.52 \mathrm{~m}^{3} \mathrm{~s}^{-1}, h=0.53 \mathrm{~m}, \quad--Q / A=0.65 \mathrm{~m}^{3} \mathrm{~s}^{-1}, h=0.67 \mathrm{~m}$, --•-- $Q / A=0.824 \mathrm{~m}^{3} \mathrm{~s}^{-1}, h=0.93 \mathrm{~m}$

pronounced deflection, but the drag per unit bed surface decreases because the number of plants per unit bed surface is lower.

\section{Drag resistance, mean motion and turbulent shear stress}

In Fig. 6, the ratio between the plant drag per unit bed surface and the streamwise momentum component, $r_{\tau}=f v / \rho g S_{b} R_{h}$, is reported for the six runs as a function of the relative flow depth $h / h_{p}$. In Fig. 7 the measured drag coefficient as a function of the plant Reynolds number $R e_{p}=d_{p} U / v$ is reported, where $d_{P}$ is the mean trunk diameter. It has to be pointed out that, in the case of willows fully submerged by the flow (i.e., one point corresponding to the higher Reynolds number for each vegetation density), the reference velocity for the evaluation of $R e_{p}$ is assumed as evaluated by the application of the DAM to the velocity profiles measured in the vegetated layer. It can be observed that, for the considered vegetation densities, $r_{\tau}$ ranges between about 0.5 and 0.95 , with the higher values for the higher vegetation density. Therefore, at least for the sparse configuration, the plants drag is of the same order of magnitude as the flow resistance due to shear at the bed and the latter is not negligible. This circumstance clashes with the assumption usually made in many simple models proposed in literature for the prediction and parameterization of a velocity profile in flows with submerged vegetation (see e.g., Kouwen et al. 1969, Christensen 1985, Raupach et al. 1996, Klopstra et al. 1997, Stephan and Gitknecht 2002, Righetti and Armanini 2002, Katul et al. 2002, Carollo et al. 2002), for which the bed shear can be considered negligible with respect to the plants drag. 


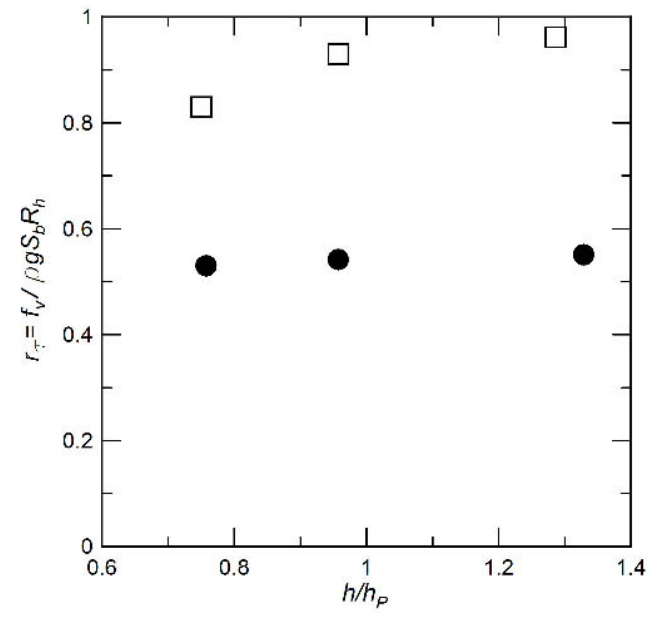

Fig. 6. Ratio between the plant drag per unit bed surface and streamwise momentum component for dense $(\square)$ and sparse $(\bullet)$ configurations.

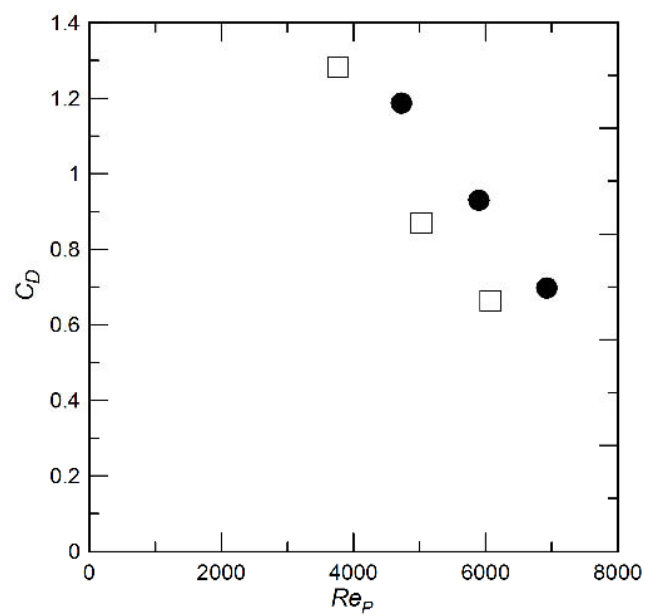

Fig. 7. Plant drag coefficient as a function of the plant Reynolds number $\operatorname{Re}_{P}=d_{P} U / v$, for dense $(\square)$ and sparse $(\bullet)$ configurations.

Moreover, the drag coefficient reported in Fig. 7 shows a strong dependence on the flow velocity (that is the plants Reynolds number), due to the plant elasticity (see, Armanini et. al. 2005) and a slight dependence on the density of vegetation. The dependence on vegetation density can be ascribed also to the mutual interaction of plants, that is, sheltering effect and to the modifications in the turbulent structure of the flow field induced by surrounding plants. Indeed, this reduction on the drag coefficient has already been investigated for rigid cylinders and has been ascribed to the wake interaction between adjacent cylinders (see e.g., Lindner 1982, Nepf 1999). Cor- 
rective procedures have been proposed for arrays of rigid cylinders, in order to correct the $C_{D}$ values (Pasche and Rouvè 1985, Nepf 1999), the applicability of these procedures to the case of flexible plants with significant bending is an open question that goes beyond the aim of the present paper.

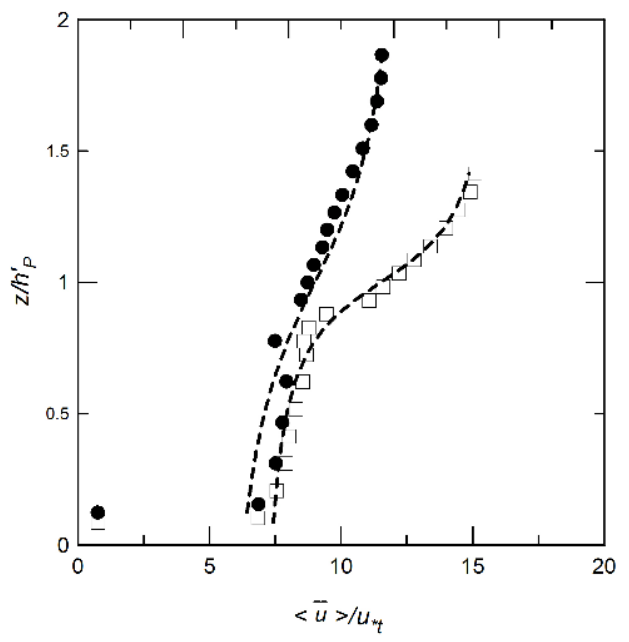

Fig. 8. Double-averaged dimensionless profiles. Free surfaces are located at $h / h_{P}^{\prime}=2.07$ and 1.55 for dense ( $\square$ ) and sparse ( $\bullet$ configurations. Dashed lines: inverse tangent formula, eq. (8) with suitable choice of the parameters $a_{i}, b_{i}$ appearing in eq. (8).

(a)

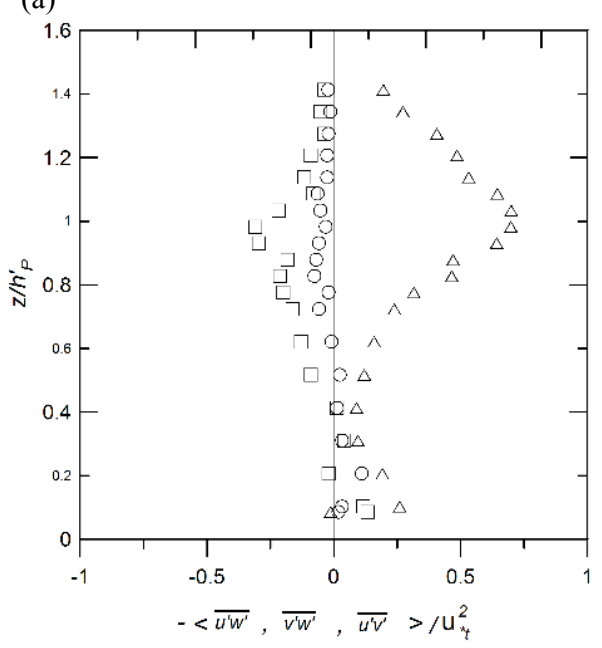

(b)

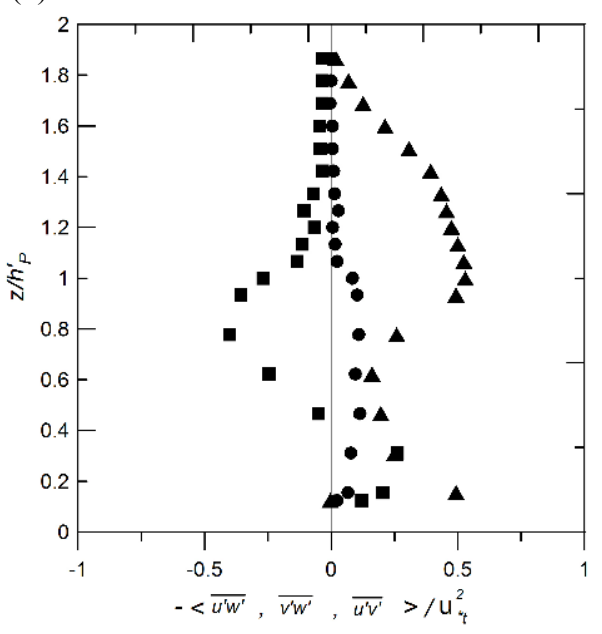

Fig. 9. Spatially averaged turbulent Reynolds profiles for dense and sparse configurations. (a) Dense configuration: $\left.\Delta \overline{\left\langle u^{\prime} w^{\prime}\right.}\right\rangle / u_{*_{t}}^{2}, \square\left\langle\overline{u^{\prime} v^{\prime}}\right\rangle / u_{*_{t}}^{2}, \circ\left\langle\overline{\left\langle v^{\prime} w^{\prime}\right.}\right\rangle / u_{*_{t}}^{2}$. (b) Sparse configuration: $\boldsymbol{\Delta}\left\langle\overline{u^{\prime} w^{\prime}}\right\rangle / u_{*_{t}}^{2}$, $\left\langle\overline{u^{\prime} v^{\prime}}\right\rangle / u_{*_{t}}^{2}, \bullet\left\langle\overline{v^{\prime} w^{\prime}}\right\rangle / u_{*_{t}}^{2}$. 
The spatially averaged dimensionless mean velocity profiles for the sparse and dense configurations are reported in Fig. 8; Figure 9 shows the spatially averaged turbulence shear stresses profiles, while Fig. 10 shows the corresponding form-induced components. In the following the height of bended vegetation $h_{P}^{\prime}$ and the shear velocity at the top of vegetation, $u_{*_{t}}=g S_{b}\left(h-h_{P}^{\prime}\right)$, are chosen as length and velocity scales in order to make the plots dimensionless. The ratio between the flow depth and the vegetation height ranges between 1.55 and 2.07, corresponding to low relative submergence. Following the definition of Nikora et al. (2001), the studied flows can be classified as a flow type II. The double-averaged velocity distributions are qualitatively similar for both flows: with an almost constant sublayer close to the free surface, a quasi linear distribution in the central region of the non-vegetated layer, a strong gradient close to the vegetation crests, the gradient being stronger for dense configuration, and an almost uniform distribution in the vegetated layer with a decreasing mean value for increasing vegetation density. Finally, closer to the bed the velocity tends to decrease due to the no-slip condition at the bed.

(a)

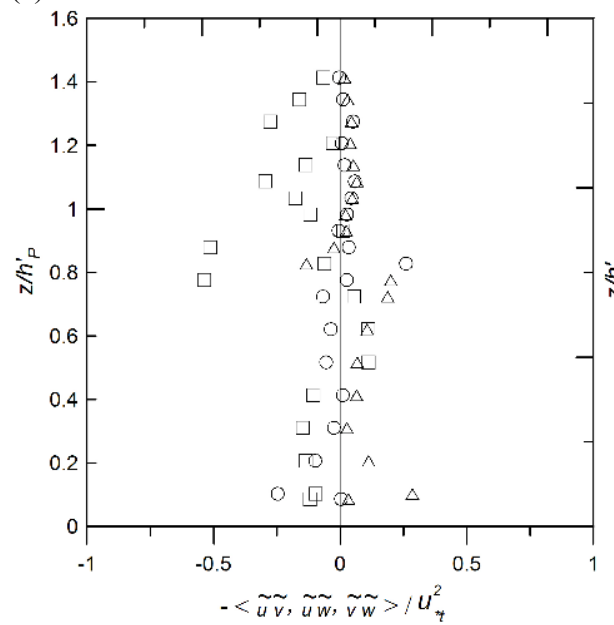

(b)

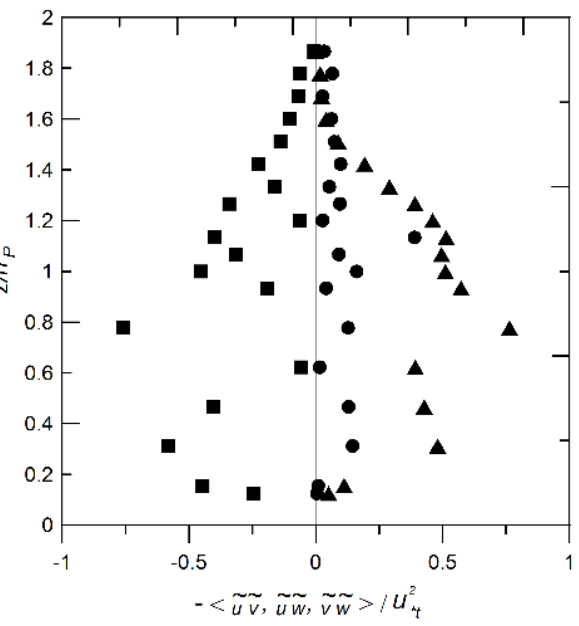

Fig. 10. Form-induced turbulent Reynolds profiles for dense and sparse configurations. (a) Dense configuration: $\Delta\langle\tilde{u} \tilde{w}\rangle / u_{*_{t}}^{2}, \square\langle\tilde{u} \tilde{v}\rangle / u_{*_{t}}^{2}, \bigcirc\langle\tilde{v} \tilde{w}\rangle / u_{*_{t}}^{2}$.

(b) Sparse configuration: $\boldsymbol{\Delta}\langle\tilde{u} \tilde{w}\rangle / u_{*_{t}}^{2}, \boldsymbol{\square}\langle\tilde{u} \tilde{v}\rangle / u_{*_{t}}^{2}, \boldsymbol{\bullet}\langle\tilde{v} \tilde{w}\rangle / u_{*_{t}}^{2}$.

The vegetated layer corresponds to the interfacial sublayer defined by Nikora et al. (2001) for low submergence flows. If $f_{P}=\langle\partial \tilde{p} / \partial x\rangle$ and $\phi\left(\left\langle\overline{u^{\prime} w^{\prime}}\right\rangle+\langle\tilde{u} \tilde{w}\rangle\right)$ in eq. (4) can be considered approximately constant along $z$ in the interfacial sublayer (wake turbulence dynamics), then a constant velocity profile can be derived (Nikora et al. 2004). Indeed, this seems to be 
just our case, because for both the runs the porosity $\phi$ appears to be constant all along the vertical and also the spatially averaged and the form-induced shear stresses profiles (Figs. 9 and 10) seem to be almost constant.

In the upper part of the vegetated layer, the constant velocity profile seems not to be valid anymore, and an inflection point can be observed close to the top of vegetation. This kind of profile is quite similar to the hyperbolic tangent profile suggested on the basis of mixing layer analogy for canopy flows by Raupach et al. (1996), for low submergence open channel flow by Katul et al. (2002) and for flows in vegetated rivers by Ghisalberti and Nepf $(2002,2004)$. Therefore, the experimental data have been compared with the following theoretical profile suggested by Ghisalberti and Nepf (2002):

$$
\langle\bar{u}\rangle=U_{0}+\frac{\Delta U}{2} \tanh \left(\frac{z-z_{i}}{2 \theta}\right),
$$

where $U_{0}=\left(U_{1}+U_{2}\right) / 2, U_{1}$ and $U_{2}$ are the lowest and highest values of flow velocity, in the present case the mean velocity values in the vegetated layer and in the almost constant sublayer at the free surface, respectively, $\Delta U=U_{2}-U, z_{i}$ is the distance from the bed where $\langle\bar{u}\rangle=U_{0}$ and $\theta$ is the momentum thickness defined as (Rogers and Moser 1994, Ghisalberti and Nepf 2002):

$$
\theta=\int_{-\infty}^{+\infty}\left(\frac{1}{4}-\frac{\langle\bar{u}\rangle-U_{0}}{\Delta U}\right) \mathrm{d} z .
$$

The momentum thickness has been determined by solving the integral of eq. (7) as a summation.

Figure 11 shows the velocity profiles made dimensionless with the mixing layer parameters. Concerning the upper layer, it can be noted that the experimental data seem to be fairly well described by the hyperbolic tangent model. However, when the lower layer is considered, the theoretical profiles tend to deviate from the experimental data, especially for the data concerning the sparse configuration.

In the same way, an inverse tangent profile (eq. 8), as proposed by $\mathrm{Ca}$ rollo et al. (2002), with suitable choice of $a_{i}$ and $b_{i}$ coefficients appearing in eq. (8), seems to successfully approximate the experimental data in the upper non-vegetated layer. Again, in the vegetated layer the theoretical profiles slightly deviate from the data, the higher differences occurring for the sparse configuration and close to the bed (Fig. 8).

$$
\frac{\langle\bar{u}\rangle}{u_{*_{t}}}=b_{0}+b_{1} \arctan \left(\frac{\left(z / h_{P}^{\prime}\right)-a_{1}}{a_{2}}\right) .
$$

In order to better understand the features of the dynamical interaction between the willows and the flow, the analysis of the turbulent second and 
third order velocity moments have been performed; the main results are reported in the following.

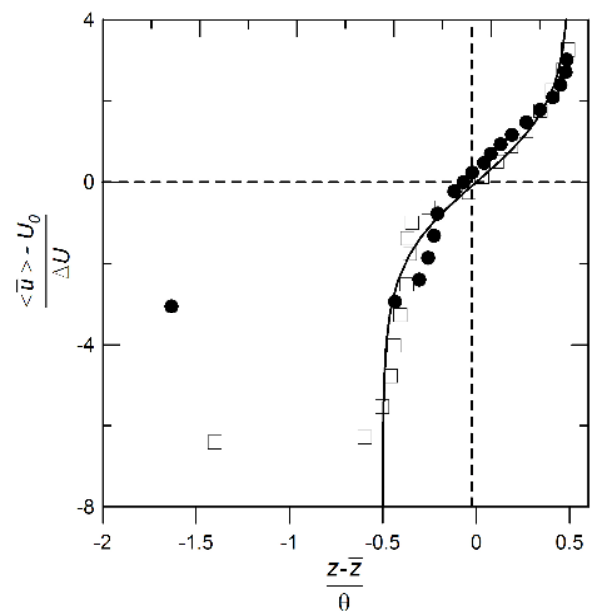

Fig. 11. Spatially averaged velocity dimensionless profiles with mixing layer variables. $\square$ Dense configuration, $\bullet$ sparse configuration; Solid line: hyperbolic tangent (eq. 6).

The spatially averaged Reynolds stress profile $-\left\langle\overline{u^{\prime} w^{\prime}}\right\rangle / u_{*_{t}}$ reported in Fig. 9 tends to assume an almost triangular shape in the upper non vegetated layer for both the configurations, with a maximum close to the top of the vegetated layer, and the maximum value for the denser vegetation. Further down into the vegetated layer, the profile tends to decrease up to a minimum in the middle of the layer and then slightly increases toward the bed.

The form-induced contributions to the Reynolds stresses are of the same order of magnitude as the spatially averaged ones for sparse configuration (Fig. 10a, b). For the dense configuration they are smaller than the spatially averaged ones but still non negligible. Moreover, while in the sparse configuration the form-induced shear stress component $-\langle\tilde{u} \tilde{w}\rangle$ is positive all along the flow depth, in the dense configuration it becomes negative (upward form-induced momentum flux) in a small region close to the top of the vegetation. This circumstance has already been reported in literature for a vegetated layer simulated by rigid cylinders (Poggi et al. 2004) and explained in (Pokrajac et al. 2008) using experiments with transverse bars. The authors ascribed this upward form-induced momentum flux to the vortex shedding phenomena that take place at the top of the roughness elements. Nevertheless, the changing sign for the denser configuration is apparently contradictory; the measurements of Poggi et al. (2004) and of Pokrajac et al. (2008) show an increase of the upward form-induced momentum flux for decreasing roughness element density, while the contrary is shown in Fig. 10. 
The explanation lies in the effect of plants flexibility. The streamlined shape assumed by the plants in the sparse configuration tends to reduce the flow separation and vortex shedding in the region close to the top of vegetation. On the other hand, in the dense configuration willows are less bent by the flow; thus, the vortex shedding is enhanced at the crests, giving as a result a net upward form-induced momentum flux in that region. Whether the different hydrodynamic behaviour between the sparse and dense configurations could also explain the different attitude to obey the mixing layer analogy is not clear. An analysis of the turbulence intensities - which will be developed in the next section - could be helpful to this purpose.

\section{Turbulent intensities}

Figure 12 shows the spatially averaged turbulence intensity profiles and Fig. 13 the corresponding dispersive components. It can be noted that in the lower region of the vegetated layer the transversal component of the spatial averaged turbulent intensity is higher than the streamwise one while they are almost equal in the upper region of the vegetated layer. This effect can be ascribed to the Karman wake generation behind trunks and branches, which tends to enhance the transversal fluctuations, especially for the dense configuration. The comparison between the spatially averaged and the forminduced turbulent intensity profiles clearly shows that the contribution of the latter to the turbulent energy budget cannot be neglected. The values of the form-induced transversal and longitudinal components are higher in the vegetated layer than in the upper layer. The registered higher values in the

(a)

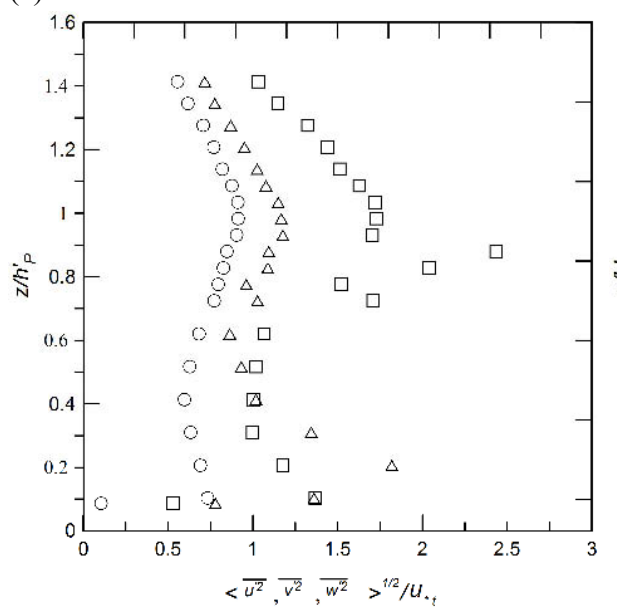

(b)

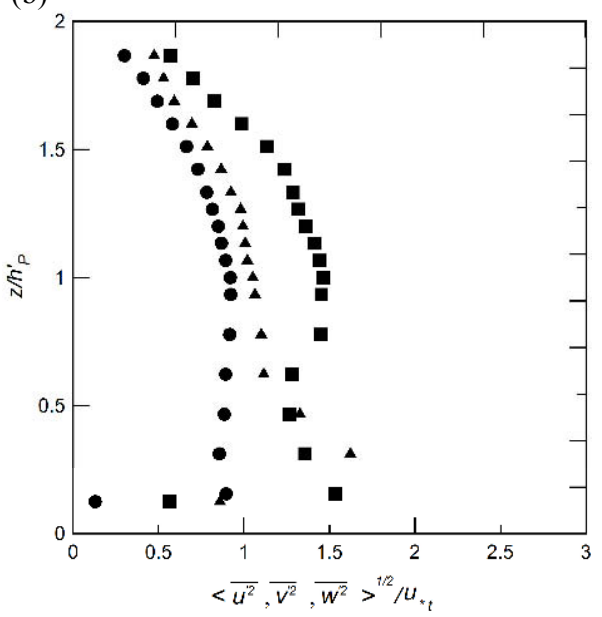

Fig. 12. Spatially averaged turbulent intensity components profiles for dense and sparse configurations.
(a) Dense configuration:
$\square\left\langle\overline{u^{\prime 2}}\right\rangle^{1 / 2} / u_{*_{t}}$
$\Delta\left\langle\overline{v^{\prime 2}}\right\rangle^{1 / 2} / u_{* t}$,
$\left.\bigcirc \overline{\left\langle w^{\prime 2}\right.}\right\rangle^{1 / 2} / u_{*_{t}}$.

(b) Sparse configuration:

$\boldsymbol{\Delta}\left\langle\overline{v^{\prime 2}}\right\rangle^{1 / 2} / u_{*_{t}}$

- $\left\langle\overline{\left.w^{\prime 2}\right\rangle^{1 / 2} / u_{*_{t}}}\right.$. 

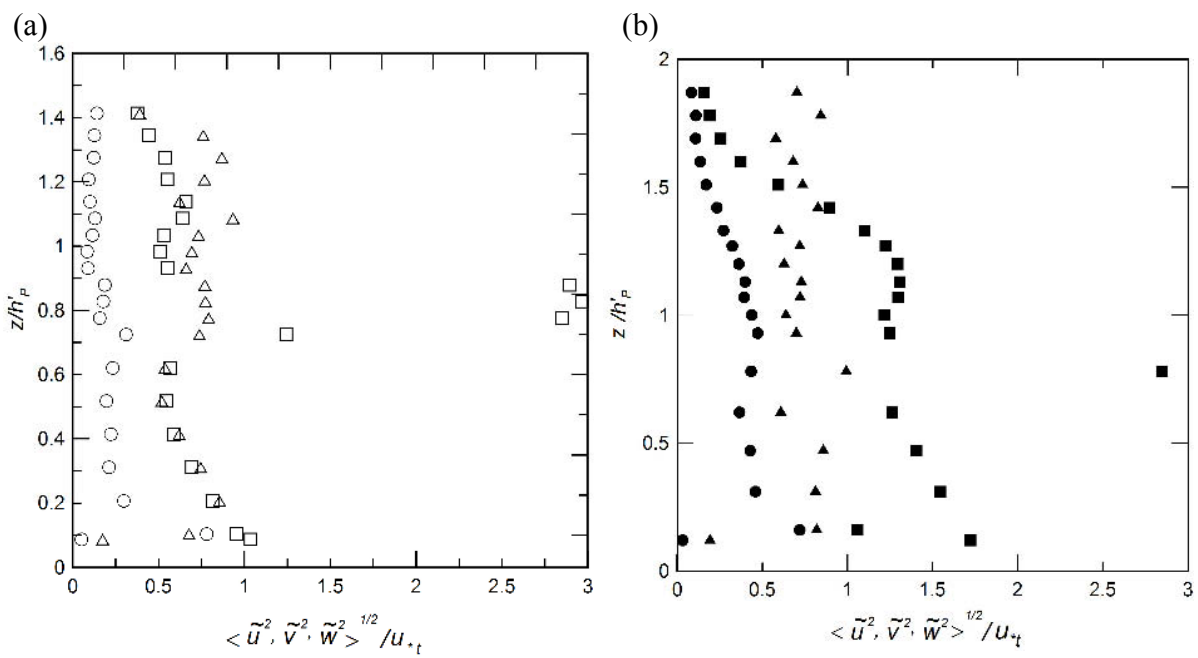

Fig. 13. Form-induced turbulent intensity components profiles for dense and sparse configurations.
(a) Dense configuration:
$\square\left\langle\tilde{u}^{2}\right\rangle^{1 / 2} / u_{*_{t}}, \Delta\left\langle\tilde{v}^{2}\right\rangle^{1 / 2} / u_{*_{t}}, \bigcirc\left\langle\tilde{w}^{2}\right\rangle^{1 / 2} / u_{*_{t}}$.
(b) Sparse configuration:
$\left\langle\tilde{u}^{2}\right\rangle^{1 / 2} / u_{* t}$,
$\boldsymbol{\Delta}\left\langle\tilde{v}^{2}\right\rangle^{1 / 2} / u_{*_{t}}$
○ $\left\langle\tilde{w}^{2}\right\rangle^{1 / 2} / u_{* t}$.

vegetated layer are a result of deviations induced by the presence of trunks and branches on the local (time averaged) motion with respect to the mean (spatially and temporally averaged) motion.

\section{Higher order turbulence moments}

Figure 14 shows the vertical profiles of the spatially averaged longitudinal third order moment $\left\langle\overline{u^{\prime 3}}\right\rangle / u_{*_{t}}^{3}$ and the corresponding form-induced contribution. Figure 15 shows the vertical profile of the third order moment $\left.\overline{\left\langle u^{\prime 2} w^{\prime}\right.}\right\rangle / u_{*_{t}}^{3}$, which is related to the transport of turbulent energy (Raupach and Shaw 1982), and the corresponding form-induced contribution.

Both plots show that above the vegetation layer the form-induced contribution is significantly lower than the spatially averaged. On the contrary, in the vegetated layer the form-induced third order moments tend to increase due to the trunks and branches effect just mentioned in the previous paragraph.

The joint analysis of the spatially averaged third order moments deserve particular attention. Indeed, flow regions dominated by ejection turbulent events are associated with $\left\langle\overline{u^{\prime 3}}\right\rangle<0$ together with $\left.\overline{\left\langle u^{\prime 2} w^{\prime}\right.}\right\rangle>0$ values. Flow regions dominated by sweep turbulent events are associated with $\left\langle\overline{u^{\prime 3}}\right\rangle>0$ together with $\left\langle\overline{u^{\prime 2} w^{\prime}}\right\rangle<0$ values instead. Therefore, looking at Figs. 14 and 15 it can be deduced that: 
(a)

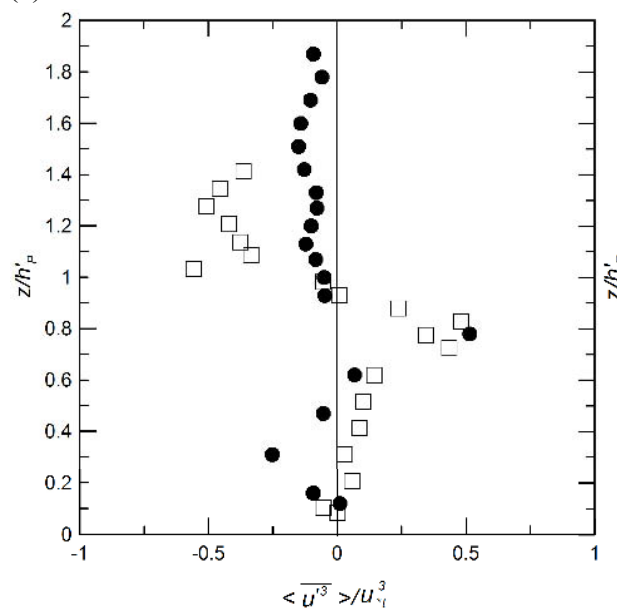

(b)

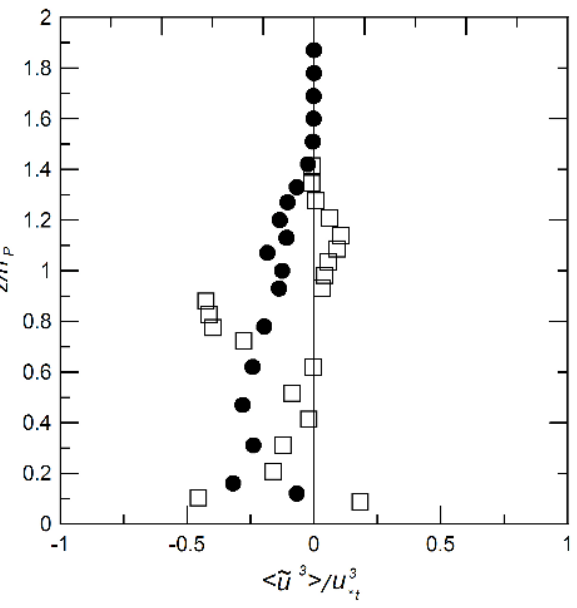

Fig. 14. Streamwise velocity third-order moment profiles for dense ( $\square$ ) and sparse $(\bullet)$ configurations: (a) spatially averaged contribution $\left\langle\overline{\left\langle u^{\prime 3}\right.}\right\rangle / u_{*_{t}}^{3}$; (b) forminduced contribution $\left\langle\tilde{u}^{3}\right\rangle / u_{* t}^{3}$.

(a)

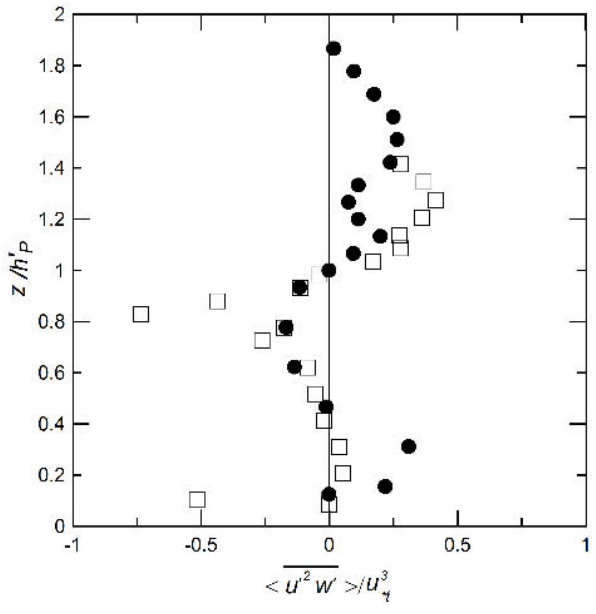

(b)

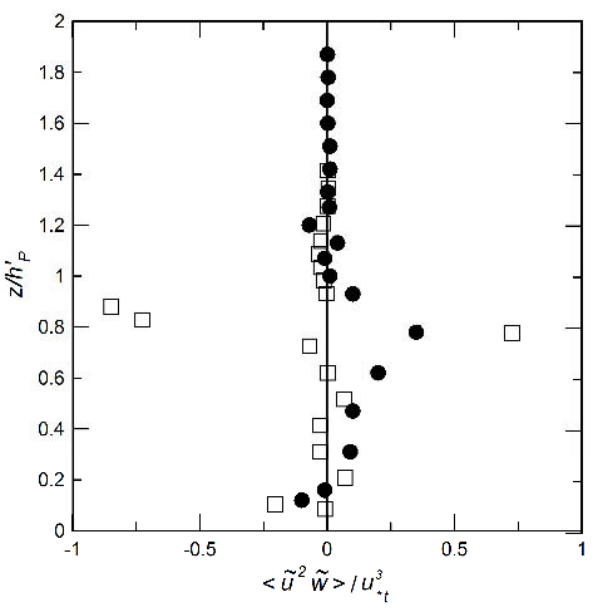

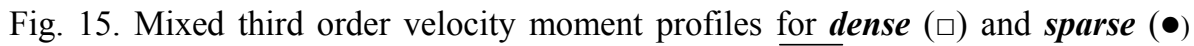
configurations: (a) spatially averaged contribution $\overline{\left\langle u^{\prime 2} w^{\prime}\right\rangle} / u_{*_{t}}^{3}$; (b) form-induced contribution $\left\langle\tilde{u}^{2} \tilde{w}\right\rangle / u_{*_{t}}^{3}$.

- For the dense configuration, the upper non vegetated layer is mainly affected by ejection events. The most part of the vegetated layer is affected by sweep events. This is a typical characteristic of a mixing layer dynamics, as also reported by Ghisalberti and Nepf (2002): the roller type vortices that 
develop are responsible for strong sweep events in the region just below the top of vegetation and for ejection events in the upper region.

- For the sparse configuration the scenario is more complicated. The upper region of the non vegetated layer is mainly dominated by ejection events down to about $z / h_{P}^{\prime} \approx 1.3$. In the vegetated layer the sweeps-dominated region is limited only to the vegetation crests proximity, while in the lower half of the vegetated layer the ejection events tend to prevail.

These considerations concur to confirm the validity of the mixing layer analogy for the dense configuration while for the sparse configuration this similitude is not fully confirmed by the experiments.

In any case, the flow region above the plants is clearly dominated by ejection turbulent events, presumably due to the dynamics of coherent eddies which take place at the top of the plants (see, e.g., Raupach et al. 1996). The features of these coherent structures in the dense and sparse configurations can presumably differ, as suggested by the third order moments analysis, but after all they are responsible for the vertical transport of momentum and substances, such as nutrients, oxygen and sediment, between the vegetation layer and the outer flow (Ikeda and Kanazawa 1996, Ghisalberti and Nepf 2005). Focusing our attention on the transport by suspension of fine sediments, it is indeed well known that ejections are responsible for the maintaining into suspension of particles heavier than fluid, supplying the required net turbulent upward momentum flux (see, e.g., Sumer and Deigaard 1981, Kaftori et al. 1995, Lu and Willmart 1973, Righetti and Romano 2004). The impact of plants on the suspended sediment transport capacity can be therefore investigated generalizing the approach proposed by $\mathrm{Lu}$ and Willmart (1973) and analyzing the spatially averaged net upward turbulent momentum flux, evaluated by means of eq. (9):

$$
\langle\text { Net Upward Turbulent Momentum Flux }\rangle=\left\langle\overline{\left(w^{+}\right)^{2} T^{+}-\left(w^{-}\right)^{2} T^{-}}\right\rangle,
$$

where $w^{+}$and $w^{-}$are the vertical upward and downward instantaneous vertical velocities in the measurement point, $T^{+}$and $T^{-}$are the time intervals for which the velocity signal was upward and downward directed. The results are shown in Fig. 16. It can be noted that all along the upper non-vegetated layer the momentum flux is positive, but the maximum values tend to be reduced as the density of plants increases. On the other hand, the vegetated layer is mainly dominated by negative momentum flux which is associated to sweep events. The negative momentum flux is stronger for the dense configuration. Therefore, it can be argued that as the vegetation density increases, the suspended sediment transport capacity of the upper non vegetated flow tends to be damped. At the same time the vegetated layer tends to entrap the suspended sediments transported by the flow, and the entrapment efficiency increases for increasing vegetation density. 


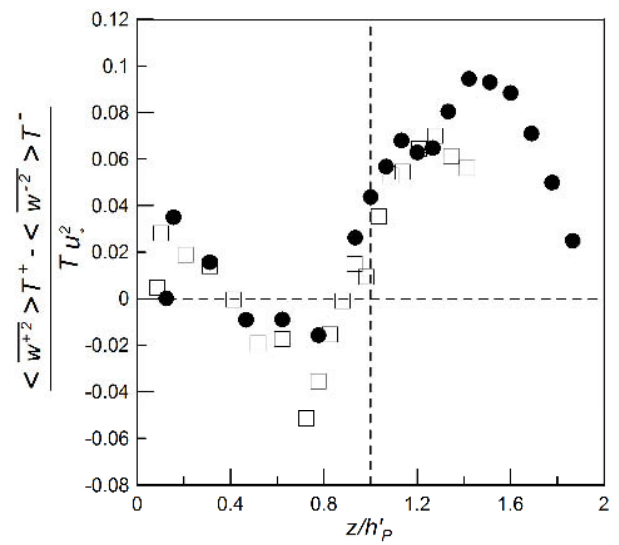

Fig. 16. Net Turbulent Upward Momentum Flux profiles for dense ( $\square$ ) and sparse $(\bullet)$ configurations.

\section{CONCLUSIONS}

An experimental study has been conducted using natural, flexible living willows to investigate the vegetation resistance for unsubmerged and submerged conditions at uniform flow condition. Real bushes, about $0.7 \mathrm{~m}$ high, have been used as vegetation, and the plants were uniformly distributed at the bed of a laboratory flume at two vegetation densities, named sparse and dense configurations. The sparse configuration reproduced the plants density and morphological features of natural willows (salix pentandra) as measured along the banks of some restored mountain streams in Trentino Region (Italy). The drag exerted to the flow by the bushes has been measured by means of a force transducer, and the plants bending has been evaluated by image analysis technique. For two experiments with fully submerged bowed plants, velocity measurements were performed and analyzed by means of the Double-Averaging Method. The following conclusion can be drawn by the analysis of the drag and velocity data:

- The plants drag per unit bed surface is of the same order of magnitude as the shear resistance at the bed, and the latter is not negligible. For a given discharge the drag per unit bed surface tends to increase with vegetation density, while the drag exerted by a single willow decreases.

- The form-induced Reynolds stresses and turbulence intensity contributions are not negligible with respect to the corresponding spatially averaged contributions.

- The average velocity, the vegetation density and the plant flexibility seem to be driving parameters for the development of a mixing layer analogy for the upper region of the flow field. This aspect has been confirmed both by the double-averaged velocity profiles and by the analysis of the double- 
averaged third order turbulent moments $\left\langle\overline{u^{\prime 3}}\right\rangle,\left\langle\overline{u^{\prime 2} w^{\prime}}\right\rangle$. The mixing layer similitude seems to be applicable only to a limited portion of the vegetated layer, located toward the plants crests. The thickness of this region decreases as the plants density decreases.

a For the lower portion of the vegetated layer, the analysis of the mean velocity profiles and of the spatial averaged and form-induced shear stresses, $\left[\left\langle\overline{u^{\prime} w^{\prime}}\right\rangle+\langle\overline{\tilde{u} \tilde{w}}\rangle\right]$ confirms the applicability of an almost constant velocity profile.

a The analysis of the spatially averaged Net Upward Turbulent Momentum Flux shows that the upper non vegetated region is dominated by ejection events, while in the vegetated layer the sweep events tend to prevail. Once more the vegetation density influences also the turbulent momentum flux dynamics. As the vegetation density increases, the Net Upward Turbulent Momentum Flux and then the related suspended sediment transport capacity in the upper layer decreases. In the vegetated layer, the negative momentum flux increases for increasing vegetation density and so increases the attitude of the vegetated layer to act as a sink for the sediments in suspension transported by the flow.

\section{References}

Armanini, A., M. Righetti, and P. Grisenti (2005), Direct measurements of vegetation resistance in prototipe scale, J. Hydraul. Res. 43, 5, 481-487.

Carollo, F.G., V. Ferro, and D. Termini (2002), Flow velocity measurements in vegetated channels, J. Hydraul. Eng. ASCE 127, 7, 664-673.

Christensen, B.A. (1985), Open channel and sheet flow over flexible roughness, Proceedings of the 21st IAHR Congress in Melbourne, Australia, 462-467.

Fathi-Maghadam, M., and N. Kouwen (1997), Nonrigid, nonsubmerged, vegetative roughness on floodplains, J. Hydraul. Eng. ASCE 123, 1, 51-57.

Finnigan, J. (2000), Turbulence in plant canopies, Ann. Rev. Fluid Mech. 32, 519-571.

Freeman, G.E., W. Rahmeyer, and R.R. Copeland (2000), Determination of resistance due to shrubs and woody vegetation, Technical Report TA7 E8, Stock Number AD A383 997 DTIC, U.S. Army Corps of Engineers, Waterways Experiment Station, Vicksburg, MS.

Ghisalberti, M., and H. Nepf (2002), Mixing layers and coherent structures in vegetated aquatic flow, J. Geophys. Res. 107, C2, 1-11.

Ghisalberti, M., and H. Nepf (2004), The limited growth of vegetated shear-layers, Water Resour. Res. 40, W07502, DOI: 10.1029/2003WR002776.

Ghisalberti, M., and H. Nepf (2005), Mass transfer in vegetated shear flows, Environ. Fluid Mech. 5, 6, 527-551, DOI: 10.1007/s10652-005-0419-1. 
Ikeda, S., and M. Kanazawa (1996), Three-dimensional organized vortices above flexible water plants, J. Hydraul. Eng. ASCE 122, 634-640.

Jarvela, J. (2002), Flow resistance of flexible and stiff vegetation: a flume study with natural plants, J. Hydrol. 269, 44-54(11).

Kaftori, D., G. Hetsroni, and S. Banerjee (1995), Particle behavior in the turbulent boundary layer: I. Motion, deposition and entrainment, Phys. Fluids 7, 5, 1095-1106.

Katul, G.G., P. Wiberg, J.D. Albertson, and G.W. Hornberger (2002), A mixing layer theory for flow resistance in shallow streams, Water Resour. Res. 38, 11 , art. no. 1250.

Klopstra, D., J.M. Barneveld, and E.H. van Noortwijk (1997), Analytical model for hydraulic roughness of submerged vegetation. Managing water: Coping with scarcity and adbundance, Proceedings of XXVII AIHR Conference, San Francisco, CA USA, August 1997.

Kouwen, N., T.E. Unny, and H.M. Hill (1969), Flow retardance in vegetated channels, J. Irrigation and Drainage Division ASCE, 95, IR2, 329-342.

Lindner, K. (1982), Der Strömungswiderstand von Pflanzenbeständen, Mitteilungen Leichtweiss-Institut für Wasserbau, TU Braunschweig, Heft 75.

Lopez, F., and M.H. García (2001), Open-channel flow through simulated vegetation: mean flow and turbulence modeling, J. Hydraul. Eng. 127, 392-402.

Lu, S.S., and W.W. Willmarth (1973), Measurement of structure of the Reynolds stress in a turbulent boundary layer, J. Fluid Mech. 60, 481-511.

Ming, R.H., and H.W. Shen (1973), Effect of tall vegetations on flow and sediment, J. Hydraul. Res. ASCE 99, HY5, 793-814.

Nepf, H.M. (1999), Drag, turbulence and diffusion in flow through emergent vegetation, Water Resour. Res. 35, 479-489.

Nepf, H.M., and E.R. Vivoni (1999), Turbulence structure in depth-limited vegetated flows: Transition between emergent and submerged regimes, Proceedings of the XXVIII AIHR Conference, Graz, Austria, September 1999.

Nikora, V., D. Goring, I. McEwan, and G. Griffiths (2001), Spatially averaged openchannel flow over rough bed, J. Hydraul. Eng. ASCE 127, 2, 123-133.

Nikora, V., K. Koll, I. McEwan, S. McLean, and A. Dittrich (2004), Velocity distribution in the roughness layer of rough-bed flows, J. Hydraul. Eng. ASCE 130, 10, 1036-1042.

Nobile, I. (2007), Dendrometric analysis of riparian vegetation in mountain streams (in Italian), Ph.D. Thesis, University of Trento, Italy.

Oplatka, M. (1998), Stabilität von Weidenverbauungen an Flussufern, Mitteilungen der Versuchsnastalt für Wasserbau, Hydrologie und Glaziologie 156.

Pasche, E., and G. Rouvé (1985), Overbank flow with vegetatively roughened flood plains, J. Hydraul. Eng. 111, 9, 1262-1278.

Petryk, S., and G. Bosmajian III (1975), Analysis of flow through vegetation, J. Hydraul. Div. ASCE 101, HY7, 871-884.

Poggi, D., A. Porporato, L. Ridolfi, J.D. Albertson, and G.G. Katul (2004), The effect of vegetation density on canopy sub-layer turbulence, Bound.-Layer Meteor. 111, 565-587. 
Pokrajac, D., McEwan, and Nikora, V. (2008), Spatially averaged turbulent stress and its partitioning, Experiments in Fluids, DOI: 10.1007/s00348-008-0463-y.

Raupach, M.R., and R.H. Shaw (1982), Averaging procedures for flow within vegetation canopies, Bound.-Layer Meteor. 22, 79-90.

Raupach, M.R., J.J. Finnigan, and Y. Brunet (1996), Coherent eddies and turbulence in vegetation canopies: The mixing layer analogy, Bound.-Layer Meteor. 78, 351-382.

Righetti, M., and A. Armanini (2002), Flow resistance in open channel flows with sparsely distributed bushes, J. Hydrol. 269, 55-64.

Righetti, M., and G.P. Romano (2004), Particle-fluid interactions in a plane nearwall turbulent flow, J. Fluid Mech. 505, 93-121.

Rogers, M.M., and R.D. Moser (1994), Direct simulation of a self-similar turbulent mixing layer, Phys. Fluids A, 6, 2, 903-923.

Stephan, U., and D. Gutknecht (2002), Hydraulic resistance of submerged flexible vegetation, J. Hydrol. 269, 27-43.

Sumer, M.B., and R. Deigaard (1981), Lift forces on moving particles near boundaries, Part 2, J. Fluid Mech. 109, 311-337.

Wilson, N.R., and R.H. Shaw (1977), A higher order closure model for canopy flow, J. Appl. Meteorol. 16, 1197-1205.

Received 19 September 2007

Accepted 12 May 2008 